\title{
Analysis of a Class of Superconvergence Patch Recovery Techniques for Linear and Bilinear Finite Elements
}

\author{
Bo $\mathrm{Li}^{*} \quad$ Zhimin Zhang ${ }^{\dagger}$
}

April 22, 2003

\begin{abstract}
Mathematical proofs are presented for the derivative superconvergence obtained by a class of patch recovery techniques for both linear and bilinear finite elements in the approximation of second order elliptic problems.
\end{abstract}

Keywords: finite element, superconvergence patch recovery, leastsquares, local projection, error estimate.

AMS Subject Classifications (1991): 65N15, 65N30.

\section{Introduction}

Recently, Zienkiewicz and Zhu have developed a superconvergence patch recovery (SPR) technique for finite element approximations of second order

*Department of Mathematics, University of California at Los Angeles, and Department of Mathematics, University of Maryland at College Park. Current address: Department of Mathematics, University of California, 405 Hilgard Avenue, Los Angeles, CA 900951555, U.S.A. E-mail: bli@math.ucla.edu. The work of this author was supported by ARPA/URI/ONR through grant N00014-92-J-1890.

${ }^{\dagger}$ Department of Mathematics, Texas Tech University, Lubbock, Texas 79409, U.S.A. E-mail: zhang@math.ttu.edu. The work of this author was partially supported by NSF through grants DMS-9622690, DMS-9626193 and DMS-9628558. 
elliptic problems $[34,35,36]$. It is a discrete version of a traditional postprocessing technique by local $L^{2}$ projection. Both techniques can be described briefly as follows. Let $u_{h}$ be a standard Galerkin finite element approximation of the exact solution $u$ of a second order elliptic problem. Let $\left(\alpha_{0}, \beta_{0}\right)$ be an interior nodal point surrounded by elements $K_{1}, \ldots, K_{m}$ of the underlying finite element mesh $\tau_{h}$ of size $h$. Set $\omega_{0}=\cup_{i=1}^{m} K_{i}$. Suppose on each element $K \in \tau_{h}, u_{h}$ belongs to the polynomial space $P(K)$ which is the restriction on $K$ of a fixed polynomial space $P$. Now two polynomials $p_{1}, p_{2} \in P\left(\omega_{0}\right)$ are determined to approximate respectively $\partial_{1} u$ and $\partial_{2} u$ on $\omega_{0}$, where $\partial_{1}=\frac{\partial}{\partial x}$ and $\partial_{2}=\frac{\partial}{\partial y}$. In the Zienkiewicz-Zhu SPR technique, $p_{1}$ and $p_{2}$ are obtained by solving the locally discrete least-squares problems

$$
\begin{aligned}
& \sum_{i=1}^{m} \sum_{j=1}^{s}\left[p_{l}\left(\xi_{i j}, \eta_{i j}\right)-\partial_{l} u_{h}\left(\xi_{i j}, \eta_{i j}\right)\right]^{2} \\
& \quad=\min _{q \in P\left(\omega_{0}\right)} \sum_{i=1}^{m} \sum_{j=1}^{s}\left[q\left(\xi_{i j}, \eta_{i j}\right)-\partial_{l} u_{h}\left(\xi_{i j}, \eta_{i j}\right)\right]^{2}, \quad l=1,2,
\end{aligned}
$$

where $\left\{\left(\xi_{i j}, \eta_{i j}\right): j=1, \ldots, s\right\}$ is a set of points in $K_{i}$ for each $i=1, \ldots, m$. In the traditional post-processing technique, $p_{1}$ and $p_{2}$ are the local $L^{2}$ projections onto $P\left(\omega_{0}\right)$ of $\partial_{1} u_{h}$ and $\partial_{2} u_{h}$, respectively,

$$
\begin{aligned}
\int_{\omega_{0}} & \left|p_{l}(x, y)-\partial_{l} u_{h}(x, y)\right|^{2} d x d y \\
& =\min _{q \in P\left(\omega_{0}\right)} \int_{\omega_{0}}\left|q(x, y)-\partial_{l} u_{h}(x, y)\right|^{2} d x d y, \quad l=1,2 .
\end{aligned}
$$

Such $p_{1}$ and $p_{2}$ are often found to be superconvergent to $\partial_{1} u$ and $\partial_{2} u$, respectively, on a set of points, $S_{i}$, in each element $K_{i}, i=1, \ldots, m$. Solve the equation (1) or (2) for all interior nodes of the mesh. If there are enough recovered superconvergence points on each element, we can then obtain by interpolation two globally continuous functions $G_{1}$ and $G_{2}$ whose restrictions on each element $K \in \tau_{h}$ are in $P(K)$ such that $G_{1}$ and $G_{2}$ globally superconverge to $\partial_{1} u$ and $\partial_{2} u$, respectively, except possibly on a boundary layer of size $h$.

We call (cf. $[34,35])\left(\alpha_{0}, \beta_{0}\right)$ a patch assembly point, $\omega_{0}$ the element patch surrounding $\left(\alpha_{0}, \beta_{0}\right),\left(\xi_{i j}, \eta_{i j}\right), j=1, \ldots, s$, the least-squares sampling points of the element $K_{i}, i=1, \ldots, m$, and the points in $S_{i}, i=1, \ldots, m$, the recovered derivative superconvergent points. We also call $p_{1}, p_{2} \in P\left(\omega_{0}\right)$ 
the recovered derivatives by the locally discrete least-squares if problem (1) is solved or the recovered derivatives by the local $L^{2}$ projection if problem (2) is solved.

Numerical experiments by Zienkiewicz and Zhu [34] have shown the derivative superconvergence for various types of finite elements in the case of locally discrete least-squares recovery but only for lower order finite elements in the case of local $L^{2}$ projection recovery. The ultraconvergence (superconvergence of order two) by the locally discrete least-squares recovery for quadratic or biquadratic elements discovered in these experiments is especially of mathematical interest. In practice, both of the recovery techniques are cost effective because of the locality of their treatment. Such techniques, especially the Zienkiewice-Zhu discrete least-squares based SPR, are also applicable to the design of a robust error estimator for the adaptive finite element method because of the global superconvergence of the recovered derivatives [33, 35, 36].

We notice that a different kind of superconvergence recovery technique for finite element approximations based on correction by interpolation has been mathematically developed by Lin, Yan, Yang, and Zhou [15, 16, 17, 18, $19,29,30]$. Both this interpolation based local correction technique and the least-squares based patch recovery technique are economic and practical, and both of them recover, in most practical cases, the global superconvergence. A common feature of these two classes of techniques is that the recovered derivatives of a finite element solution lie in a space of piecewise polynomials same as or even larger than that the solution itself lies in. We refer to the recent work $[1,2,3,4]$ for a series of studies in a computer based approach on the superconvergence for finite element approximations.

Mathematical analysis for the superconvergence patch recovery techniques first appear in $[25,27]$ for the recovered derivatives by the locally discrete least-squares for one-dimensional problems. Generalization to two-dimensional tensor product finite elements has been made in [26, 28].

In this paper, we consider both the locally discrete least-squares recovery and the local $L^{2}$ projection recovery for both triangular linear and rectangular bilinear finite element approximations of general second order elliptic problems on two-dimensional convex polygonal domains. We prove that the derivative superconvergence is achieved by both of the methods for the triangular linear element on a strongly regular family of meshes, by the locally discrete least-squares recovery for the rectangular bilinear element on a quasi-uniform family of meshes, and by the local $L^{2}$ projection recovery 
for the rectangular bilinear element on a unidirectionally uniform family of meshes. We also give an example which strongly suggests that, for the local $L^{2}$ projection recovery by the rectangular bilinear element, the recovered derivatives will not be superconvergent if the underlying family of meshes are only quasi-uniform, a phenomenon that has been in fact numerically observed in [34].

The key argument in our proofs is based on an observation on the two recovery techniques as well as an exploitation of the earlier work of the mathematical analysis on the so-called natural superconvergence for the linear and bilinear finite elements, see, e.g., [5, 6, 10, 11, 12, 13, 14, 23, 24, 31, 32]. For a uniformly regular family of meshes, the idea of such an argument is clear and the proof is almost trivial. However, difficulties arise when a more general family of meshes are considered.

We remark that we consider affine rectangular bilinear elements rather than general isoparametric quadrilateral bilinear elements. This is simply for the convenience of the exposition of the main idea. The generalization can be easily made within our framework via the work by Lin and Whiteman [13], see also [8, 26].

In Section 2, we state our main results which include local superconvergence estimates that only involve the local smoothness of the exact solution and the local regularity of the underlying family of meshes. In Section 3, we give proofs of our main results. In Section 4, we present an example in a one-dimensional setting concerning the local $L^{2}$ projection recovery.

\section{Main Results}

For the simplicity of exposition, let $\Omega$ be a convex polygonal domain in the $x y$-plane. We consider the following boundary value problem:

$$
\left\{\begin{aligned}
-\sum_{i, j=1}^{2} \partial_{j}\left(a_{i j} \partial_{i} u\right)+\sum_{i=1}^{2} b_{i} \partial_{i} u+c u & =f, & & \text { in } \Omega, \\
u & =0, & & \text { on } \partial \Omega,
\end{aligned}\right.
$$

where $a_{i j}, b_{i}, c$, and $f$ are sufficiently smooth functions defined on $\bar{\Omega}$, and

$$
\sum_{i, j=1}^{2} a_{i j}(x, y) d_{i} d_{j} \geq a_{0}\left(d_{1}^{2}+d_{2}^{2}\right), \quad \forall\left(d_{1}, d_{2}\right) \in \mathbf{R}^{2}, \quad \forall(x, y) \in \Omega
$$


for some constant $a_{0}>0$.

As usual, for an integer $k \geq 0$ and an extended real number $p$ with $1 \leq p \leq \infty, W^{k, p}(\Omega)$ and $W_{0}^{k, p}(\Omega)$ are the Sobolev spaces over the domain $\Omega$, and $\|\cdot\|_{k, p, \Omega}$ and $|\cdot|_{k, p, \Omega}$ the corresponding norms and seminorms. If $p=2$ we write $H^{k}(\Omega)$ and $H_{0}^{k}(\Omega)$ instead of $W^{k, 2}(\Omega)$ and $W_{0}^{k, 2}(\Omega)$, respectively. We define the bilinear form $A: H_{0}^{1}(\Omega) \times H_{0}^{1}(\Omega) \rightarrow \mathbf{R}$ associated with the elliptic problem (3) by

$$
A(v, w)=\int_{\Omega}\left(\sum_{i, j=1}^{2} a_{i j} \partial_{i} v \partial_{j} w+\sum_{i=1}^{2} b_{i} \partial_{i} v w+c v w\right) d x d y, \quad \forall v, w \in H_{0}^{1}(\Omega),
$$

and assume that $A$ is $H_{0}^{1}(\Omega)$-elliptic, i.e., there exists a constant $\sigma>0$ such that

$$
A(v, v) \geq \sigma\|v\|_{1,2, \Omega}^{2}, \quad \forall v \in H_{0}^{1}(\Omega) .
$$

We let $u \in H_{0}^{1}(\Omega)$ be the unique generalized solution of (3) which is defined by

$$
A(u, v)=(f, v), \quad \forall v \in H_{0}^{1}(\Omega),
$$

where $(\cdot, \cdot)$ denotes the inner product of $L^{2}(\Omega)$.

Now let $\left\{\tau_{h}: 0<h \leq h_{0}\right\}$, where $h_{0}$ is a positive constant, be a quasiuniform family of finite element meshes parameterized by the mesh size $h$ of $\tau_{h}$, covering the domain $\Omega$, i.e., $\bar{\Omega}=\cup_{K \in \tau_{h}} K$ for each $h \in\left(0, h_{0}\right]$ [9]. We consider two cases. In the first case, we assume that all elements of $\tau_{h}\left(0<h \leq h_{0}\right)$ are triangles and we consider the affine linear finite element approximation. Thus, we define for each $h \in\left(0, h_{0}\right]$ the finite element space to be

$$
V_{h}=\left\{v \in C(\bar{\Omega}):\left.v\right|_{K} \in P_{1}(K), \forall K \in \tau_{h} ; v=0 \text { on } \partial \Omega\right\},
$$

where $P_{1}(K)$ denotes the restriction on $K$ of $P_{1}$, the space of all linear polynomials. In the second case, we assume that all elements of $\tau_{h}\left(0<h \leq h_{0}\right)$ are rectangles with sides parallel to the coordinate axes, respectively, and we consider the affine bilinear finite element approximation. (We have implicitly assumed in this case that the boundary of $\Omega$ is composed of line segments parallel to the coordinate axes. This in turn implies that $\Omega$ is just a rectangular domain by its convexity.) Thus, we define for each $h \in\left(0, h_{0}\right]$ the finite element space to be

$$
V_{h}=\left\{v \in C(\bar{\Omega}):\left.v\right|_{K} \in Q_{1}(K), \forall K \in \tau_{h} ; v=0 \text { on } \partial \Omega\right\},
$$


where $Q_{1}(K)$ denotes the restriction on $K$ of $Q_{1}$, the space of all bilinear polynomials. In both cases, we have $V_{h} \subset H_{0}^{1}(\Omega), 0<h \leq h_{0}$. We also let $u_{h} \in V_{h}$ for each $h \in\left(0, h_{0}\right]$ be the unique finite element solution defined by

$$
A\left(u_{h}, v_{h}\right)=\left(f, v_{h}\right), \quad \forall v_{h} \in V_{h} .
$$

Let us fix $h \in\left(0, h_{0}\right]$ throughout the paper. The regularity of the mesh $\tau_{h}$ will always be referred to that of the whole family of meshes containing $\tau_{h}$. As in Section 1, for an interior nodal point $\left(\alpha_{0}, \beta_{0}\right)$ of $\tau_{h}$ which is surrounded by elements $K_{1}, \ldots, K_{m}$, we set the element patch $\omega_{0}=\cup_{i=1}^{m} K_{i}$. According to Zienkiewicz and Zhu [34], we choose the gravity center of element $K_{i}$, denoted by $\left(\xi_{i}, \eta_{i}\right)$, as the only sampling point of the element $K_{i}, i=1, \ldots, m$, for both the linear and bilinear finite element approximations. For convenience, we define in the sequel the polynomial space $P=P_{1}$ when considering the triangular linear element and $P=Q_{1}$ when considering the rectangular bilinear element. For the element patch $\omega_{0}$, we define the recovered derivatives to be the polynomials $p_{1}, p_{2} \in P\left(\omega_{0}\right)$ that satisfy the equation (1) which becomes in the present setting

$$
\begin{aligned}
\sum_{i=1}^{m} & {\left[p_{l}\left(\xi_{i}, \eta_{i}\right)-\partial_{l} u_{h}\left(\xi_{i}, \eta_{i}\right)\right]^{2} } \\
& =\min _{q \in P\left(\omega_{0}\right)} \sum_{i=1}^{m}\left[q\left(\xi_{i}, \eta_{i}\right)-\partial_{l} u_{h}\left(\xi_{i}, \eta_{i}\right)\right]^{2}, \quad l=1,2,
\end{aligned}
$$

for the locally discrete least-squares recovery and that satisfy the equation (2) for the local $L^{2}$ projection recovery.

In what follows, the symbol $C$ will be used as a generic constant varying with the context and will be always assumed to be independent of the solution $u$, the mesh size $h$, and the element patch $\omega_{0}$, except when the dependence is otherwise indicated.

Our first result is the existence and uniqueness of the recovered derivatives.

Lemma 1 For any element patch $\omega_{0}=\cup_{i=1}^{m} K_{i}$ surrounding a patch assembly point $\left(\alpha_{0}, \beta_{0}\right)$, both the minimization problems (4) and (2) admit unique minimizers $p_{1}, p_{2} \in P\left(\omega_{0}\right)$. Moreover, $p_{1}$ and $p_{2}$ are characterized by

$$
\sum_{i=1}^{m}\left[p_{l}\left(\xi_{i}, \eta_{i}\right)-\partial_{l} u_{h}\left(\xi_{i}, \eta_{i}\right)\right] q\left(\xi_{i}, \eta_{i}\right)=0, \quad \forall q \in P\left(\omega_{0}\right), \quad l=1,2,
$$


for the locally discrete least-squares recovery and by

$$
\int_{\omega_{0}}\left[p_{l}(x, y)-\partial_{l} u_{h}(x, y)\right] q(x, y) d x d y=0, \quad \forall q \in P\left(\omega_{0}\right), \quad l=1,2,
$$

for the local $L^{2}$ projection recovery.

We recall that a quasi-uniform triangular mesh is strongly regular if any two adjacent elements in the mesh form an approximate parallelogram in which the difference of the two vectors corresponding to any two opposite sides of the parallelogram is bounded above by $C h^{2}$, cf. $[5,6,14,32]$. Obviously, a uniform triangular mesh is always strongly regular but the reverse is not true in general. Practically, strongly regular triangular meshes can cover domains such as convex quadrilaterals that can not be covered by uniform triangular meshes.

The following theorem validates the recovered superconvergence by the triangular linear element.

Theorem 1 Suppose that the solution $u \in H_{0}^{1}(\Omega) \cap W^{3, \infty}(\Omega)$. Suppose also that the triangular mesh $\tau_{h}$ is strongly regular. Then, for any element patch $\omega_{0}=\cup_{i=1}^{m} K_{i}$ surrounding a patch assembly point $\left(\alpha_{0}, \beta_{0}\right)$, we have the following superconvergence estimate for the recovered derivatives $p_{1}, p_{2} \in P\left(\omega_{0}\right)=P_{1}\left(\omega_{0}\right)$ defined by $(4)$ or $(2)$

$$
\left|p_{l}\left(\alpha_{0}, \beta_{0}\right)-\partial_{l} u\left(\alpha_{0}, \beta_{0}\right)\right| \leq C h^{2}|\ln h|\|u\|_{3, \infty, \Omega}, \quad l=1,2 .
$$

We recall that a quasi-uniform rectangular mesh covering a rectangular domain is called unidirectionally uniform if all parallel element edges in the mesh have the same length [7]. Clearly, such a mesh is in fact a Cartesian product of two one-dimensional, uniform meshes along the $x$ and $y$ axes, respectively.

The following theorem validates the recovered superconvergence by the rectangular bilinear element. Notice that, for the local $L^{2}$ projection recovery, we need the assumption that the underlying rectangular mesh is unidirectionally uniform. An example will be given in Section 4 to strongly suggest that such a stronger regularity assumption on the mesh can not be replaced by quasi-uniformity. 
Theorem 2 Suppose that the solution $u \in H_{0}^{1}(\Omega) \cap W^{3, \infty}(\Omega)$. Suppose also that the rectangular mesh $\tau_{h}$ is quasi-uniform when considering the locally discrete least-squares recovery and is unidirectionally uniform when considering the local $L^{2}$ projection recovery. Then, for any element patch $\omega_{0}=\cup_{i=1}^{m} K_{i}$ surrounding a patch assembly point $\left(\alpha_{0}, \beta_{0}\right)$, we have the following superconvergence estimate for the recovered derivatives $p_{1}, p_{2} \in P\left(\omega_{0}\right)=Q_{1}\left(\omega_{0}\right)$ defined by (4) or (2)

$$
\left|p_{l}\left(\alpha_{0}, \beta_{0}\right)-\partial_{l} u\left(\alpha_{0}, \beta_{0}\right)\right| \leq C h^{2}|\ln h|\|u\|_{3, \infty, \Omega}, \quad l=1,2 .
$$

Now let $G_{1}$ and $G_{2}$ be the two continuous functions that are piecewise linear or bilinear defined on the union of all interior elements of $\tau_{h}$ by interpolating the two recovered derivatives at all assembly points, respectively. Directly from the above two theorems, we have the following result on the globally uniform superconvergence estimate on interior elements of $\tau_{h}$.

Corollary 1 With the same hypothesis as in Theorem 1 and Theorem 2 on the smoothness of the solution $u$ and the regularity of the mesh $\tau_{h}$, we have

$$
\max _{(x, y) \in K}\left|G_{l}(x, y)-\partial_{l} u(x, y)\right| \leq C h^{2}|\ln h|\|u\|_{3, \infty, \Omega}, \quad l=1,2
$$

for any $K \in \tau_{h}$ such that $\partial K \cap \partial \Omega=\emptyset$.

We now state the result of local estimate with regard to the local smoothness of the solution $u$ and the local regularity of the mesh $\tau_{h}$.

Theorem 3 Let $\Omega_{0}$ and $\Omega_{1}$ be two Lipschitz domains such that $\Omega_{0} \subset \subset$ $\Omega_{1} \subset \subset \Omega$. Suppose that the solution $u \in H_{0}^{1}(\Omega) \cap H^{2}(\Omega) \cap W^{3, \infty}\left(\Omega_{1}\right)$. Suppose also that the triangular mesh $\tau_{h}$ is quasi-uniform in $\Omega$ and is strongly regular in $\Omega_{1}$. Then, for any element patch $\omega_{0}=\cup_{i=1}^{m} K_{i}$ surrounding a patch assembly point $\left(\alpha_{0}, \beta_{0}\right)$ such that $\omega_{0} \subset \bar{\Omega}_{0}$, we have the following local superconvergence estimate for the recovered derivatives by the triangular linear element $p_{1}, p_{2} \in P\left(\omega_{0}\right)=P_{1}\left(\omega_{0}\right)$ defined by (4) or (2)

$$
\left|p_{l}\left(\alpha_{0}, \beta_{0}\right)-\partial_{l} u\left(\alpha_{0}, \beta_{0}\right)\right| \leq C h^{2}|\ln h|\left(\|u\|_{3, \infty, \Omega_{1}}+\|u\|_{2,2, \Omega}\right), \quad l=1,2,
$$

where the constant $C$ may depend on $\Omega_{0}$ and $\Omega_{1}$. 
Theorem 4 Let $\Omega_{0}$ and $\Omega_{1}$ be two Lipschitz domains such that $\Omega_{0} \subset \subset$ $\Omega_{1} \subset \subset \Omega$. Suppose that the solution $u \in H_{0}^{1}(\Omega) \cap H^{2}(\Omega) \cap W^{3, \infty}\left(\Omega_{1}\right)$. Suppose also that the rectangular mesh $\tau_{h}$ is quasi-uniform in $\Omega$, and is unidirectionally uniform in $\Omega_{1}$ when considering the local $L^{2}$ projection recovery. Then, for any element patch $\omega_{0}=\cup_{i=1}^{m} K_{i}$ surrounding a patch assembly point $\left(\alpha_{0}, \beta_{0}\right)$ such that $\omega_{0} \subset \bar{\Omega}_{0}$, we have the following local superconvergence estimate for the recovered derivatives by the rectangular bilinear element $p_{1}, p_{2} \in P\left(\omega_{0}\right)=Q_{1}\left(\omega_{0}\right)$ defined by (4) or (2)

$$
\left|p_{l}\left(\alpha_{0}, \beta_{0}\right)-\partial_{l} u\left(\alpha_{0}, \beta_{0}\right)\right| \leq C h^{2}|\ln h|\left(\|u\|_{3, \infty, \Omega_{1}}+\|u\|_{2,2, \Omega}\right), \quad l=1,2,
$$

where the constant $C$ may depend on $\Omega_{0}$ and $\Omega_{1}$.

A direct consequence of the above two theorems is the following result on the locally uniform superconvergence.

Corollary 2 With the same hypothesis as in Theorem 3 and Theorem 4 on the domains $\Omega_{0}$ and $\Omega_{1}$, the smoothness of the solution $u$, and the regularity of the mesh $\tau_{h}$, we have

$$
\max _{(x, y) \in K}\left|G_{l}(x, y)-\partial_{l} u(x, y)\right| \leq C h^{2}|\ln h|\left(\|u\|_{3, \infty, \Omega_{1}}+\|u\|_{2,2, \Omega}\right), \quad l=1,2,
$$

for any $K \in \tau_{h}$ such that $K \subset \bar{\Omega}_{0}$, where the constant $C$ may depend on $\Omega_{0}$ and $\Omega_{1}$.

\section{Proofs}

Proof of Lemma 1 We consider two cases.

Case 1. The locally discrete least-squares recovery. Recall that $\left(\xi_{i}, \eta_{i}\right), i=$ $1, \ldots, m$, are the gravity centers of elements $K_{i}$ in the element patch $\omega_{0}=$ $\cup_{j=1}^{m} K_{j}$ surrounding the patch assembly point $\left(\alpha_{0}, \beta_{0}\right)$. Notice that if $q \in$ $P\left(\omega_{0}\right)$ satisfies

$$
q\left(\xi_{i}, \eta_{i}\right)=0, \quad i=1, \ldots, m,
$$

then $q=0$ identically on $\omega_{0}$ for both the triangular linear element and the rectangular bilinear element. Thus, the mapping

$$
q \rightarrow\|q\| \equiv\left\{\sum_{i=1}^{m}\left[q\left(\xi_{i}, \eta_{i}\right)\right]^{2}\right\}^{\frac{1}{2}}
$$


defines a norm on the space $P\left(\omega_{0}\right)$. With this norm the nonnegative functional $F_{l}: P\left(\omega_{0}\right) \rightarrow \mathbf{R}$ defined for $l=1$ or 2 by (cf. (4))

$$
F_{l}(q)=\sum_{i=1}^{m}\left|q\left(\xi_{i}, \eta_{i}\right)-\partial_{l} u_{h}\left(\xi_{i}, \eta_{i}\right)\right|^{2}, \quad q \in P\left(\omega_{0}\right),
$$

is obviously continuous. Moreover, $F_{l}(q) \rightarrow \infty$ as $\|q\| \rightarrow \infty$. Therefore, the local compactness of the finite-dimensional space $P\left(\omega_{0}\right)$ implies the existence of minimizers of $F_{l}$ on $P\left(\omega_{0}\right)$.

It is easy to verify that the second variation of $F_{l}$ satisfies

$$
\delta^{2} F_{l}(q)(p, p)=2\|p\|^{2}>0, \quad \forall q, p \in P\left(\omega_{0}\right), p \neq 0 .
$$

Consequently, the functional $F_{l}: P\left(\omega_{0}\right) \rightarrow \mathbf{R}$ is strictly convex. This implies the uniqueness of the minimizer of $F_{l}$ on $P\left(\omega_{0}\right)$.

Now the unique minimizer $p_{l} \in P\left(\omega_{0}\right)$ of $F_{l}$ on $P\left(\omega_{0}\right)$ is characterized by

$$
\begin{aligned}
& F_{l}\left(p_{l}+t q\right)-F_{l}\left(p_{l}\right) \\
& \quad=\|q\|^{2} t^{2}+2\left\{\sum_{i=1}^{m} q\left(\xi_{i}, \eta_{i}\right)\left[p_{l}\left(\xi_{i}, \eta_{i}\right)-\partial_{l} u_{h}\left(\xi_{i}, \eta_{i}\right)\right]\right\} t \geq 0
\end{aligned}
$$

for any $q \in P\left(\omega_{0}\right)$ and any $t \in \mathbf{R}$. This is equivalent to (5).

Case 2. The local $L^{2}$ projection recovery. We can proceed the proof for this case similarly by using the $L^{2}\left(\omega_{0}\right)$ norm instead of the norm defined by (9) and by using the functional $F_{l}: P\left(\omega_{0}\right) \rightarrow \mathbf{R}$ defined by (cf. (2))

$$
F_{l}(q)=\int_{\omega_{0}}\left|q(x, y)-\partial_{l} u_{h}(x, y)\right|^{2} d x d y, \quad q \in P\left(\omega_{0}\right),
$$

instead of that defined by (10). The proof is complete.

Remark By the same argument as in the above proof, we can easily generalize Lemma 1 to obtain the existence and uniqueness for the minimization problems (1) and (2) for any type of finite element approximation. However, for the problem (1) we need to choose all the sampling points $\left(\xi_{i j}, \eta_{i j}\right) \in K_{i}(1 \leq i \leq m, 1 \leq j \leq s)$ in such a way that, for any $q \in P\left(\omega_{0}\right)$, we have $q=0$ identically on $\omega_{0}$ whenever $q=0$ at all these points $\left(\xi_{i j}, \eta_{i j}\right), 1 \leq i \leq m, 1 \leq j \leq s$.

To prove Theorem 1 we need the following result on the stability of recovered derivatives for the triangular finite element. 
Lemma 2 Suppose that the solution $u \in H_{0}^{1}(\Omega) \cap W^{3, \infty}(\Omega)$. Suppose also that the triangular mesh $\tau_{h}$ is quasi-uniform. For any element patch $\omega_{0}=$ $\cup_{i=1}^{m} K_{i}$ surrounding an assembly point $\left(\alpha_{0}, \beta_{0}\right)$, let $p_{1}, p_{2} \in P\left(\omega_{0}\right)=P_{1}\left(\omega_{0}\right)$ be the recovered derivatives defined by (4) or (2). Then, we have the stability estimate

$$
\left|\nabla p_{l}\left(\alpha_{0}, \beta_{0}\right)\right| \leq C\|u\|_{3, \infty, \Omega}, \quad l=1,2 .
$$

Proof We consider two cases separately.

Case 1. The locally discrete least-squares recovery. The mapping $p \rightarrow$ $\left|\nabla p\left(\alpha_{0}, \beta_{0}\right)\right|$ defines a seminorm on $P_{1}\left(\omega_{0}\right)$. Since $P_{1}\left(\omega_{0}\right)$ is finite-dimensional and the mesh $\tau_{h}$ is quasi-uniform, by an affine transformation from the patch $\omega_{0}$ to a reference patch $\hat{\omega}_{0}$ with diameter of size $O(1)$, we have the inverse estimate

$$
\left|\nabla q\left(\alpha_{0}, \beta_{0}\right)\right| \leq C h^{-1}\|q\|, \quad \forall q \in P_{1}\left(\omega_{0}\right),
$$

where $\|\cdot\|$ is the norm on $P_{1}\left(\omega_{0}\right)$ defined by $(9)$.

Let $L_{l} \in P_{1}\left(\omega_{0}\right)$ be the linear part of $\partial_{l} u$ around the assembly point $\left(\alpha_{0}, \beta_{0}\right)$ :

$$
L_{l}(x, y)=\partial_{l} u\left(\alpha_{0}, \beta_{0}\right)+\nabla \partial_{l} u\left(\alpha_{0}, \beta_{0}\right) \cdot\left(x-\alpha_{0}, y-\beta_{0}\right), \quad(x, y) \in \omega_{0} .
$$

Obviously, $\nabla L_{l}\left(\alpha_{0}, \beta_{0}\right)=\nabla \partial_{l} u\left(\alpha_{0}, \beta_{0}\right)$, and

$$
\left|L_{l}(x, y)-\partial_{l} u(x, y)\right| \leq C h^{2}\|u\|_{3, \infty, \omega_{0}}, \quad \forall(x, y) \in \omega_{0} .
$$

We thus have by (13) that

$$
\begin{aligned}
\left|\nabla p_{l}\left(\alpha_{0}, \beta_{0}\right)\right| & \leq\left|\nabla\left(p_{l}-L_{l}\right)\left(\alpha_{0}, \beta_{0}\right)\right|+\left|\nabla L_{l}\left(\alpha_{0}, \beta_{0}\right)\right| \\
& \leq C h^{-1}\left\|p_{l}-L_{l}\right\|+\left|\nabla \partial_{l} u\left(\alpha_{0}, \beta_{0}\right)\right| .
\end{aligned}
$$

Since $p_{l} \in P_{1}\left(\omega_{0}\right)$ is the minimizer of the functional $F_{l}: P_{1}\left(\omega_{0}\right) \rightarrow \mathbf{R}$ defined by $(10)$ on $P_{1}\left(\omega_{0}\right)$, we have

$$
\begin{aligned}
\| p_{l} & -L_{l} \| \\
& \leq\left[\sum_{i=1}^{m}\left|p_{l}\left(\xi_{i}, \eta_{i}\right)-\partial_{l} u_{h}\left(\xi_{i}, \eta_{i}\right)\right|^{2}\right]^{\frac{1}{2}}+\left[\sum_{i=1}^{m}\left|\partial_{l} u_{h}\left(\xi_{i}, \eta_{i}\right)-L_{l}\left(\xi_{i}, \eta_{i}\right)\right|^{2}\right]^{\frac{1}{2}}
\end{aligned}
$$




$$
\begin{aligned}
& \leq 2\left[\sum_{i=1}^{m}\left|L_{l}\left(\xi_{i}, \eta_{i}\right)-\partial_{l} u_{h}\left(\xi_{i}, \eta_{i}\right)\right|^{2}\right]^{\frac{1}{2}} \\
& \leq 2\left[\sum_{i=1}^{m}\left|L_{l}\left(\xi_{i}, \eta_{i}\right)-\partial_{l} u\left(\xi_{i}, \eta_{i}\right)\right|^{2}\right]^{\frac{1}{2}}+2\left[\sum_{i=1}^{m}\left|\partial_{l} u\left(\xi_{i}, \eta_{i}\right)-\partial_{l} u_{h}\left(\xi_{i}, \eta_{i}\right)\right|^{2}\right]^{\frac{1}{2}} \\
& \leq C h\|u\|_{3, \infty, \Omega},
\end{aligned}
$$

where in the last step we used (14) as well as the $W^{1, \infty}$ error estimate for the linear finite element approximation $[6,9,20,21,22,32]$. The stability estimate (12) now follows from (15) and (16) in this case.

Case 2. The local $L^{2}$ projection recovery. The proof of (12) in this case is similar to that in Case 1 except we replace the norm (9) by the $L^{2}\left(\omega_{0}\right)$ norm and the functional $F_{l}: P_{1}\left(\omega_{0}\right) \rightarrow \mathbf{R}$ defined by (10) by that defined by (11). The proof is complete.

Now we are ready to prove Theorem 1.

Proof of Theorem 1 Let $u,\left(\alpha_{0}, \beta_{0}\right), K_{i}$, and $\omega_{0}=\cup_{i=1}^{m} K_{i}$ be all the same as in the theorem. We may assume without loss of generality that $m=6$, i.e., the patch $\omega_{0}$ consists exactly of six elements $K_{i}, i=1, \ldots, 6$, since this is so with $h$ sufficiently small by the strongly regular property of the triangular finite element mesh $\tau_{h}$.

For $i=1, \ldots, 6$, we assume that the element $K_{i}$ is adjacent to the element $K_{i+1}$ and denote by $\left(\gamma_{i}, \delta_{i}\right)$ the midpoint of $\partial K_{i} \cap \partial K_{i+1}$, the common edge of the two elements $K_{i}$ and $K_{i+1}$, where we adopt the convention that two indices $i_{1}$ and $i_{2}$ are the same if and only if $i_{1} \equiv i_{2}(\bmod 6)$. Fix $l=1$ or 2 . We have by the previous works $[5,6,10,12,14,24,32]$ that

$$
\begin{gathered}
\left|\partial_{l} u\left(\gamma_{i}, \delta_{i}\right)-\frac{1}{2}\left[\left.\partial_{l} u_{h}\right|_{K_{i}}\left(\gamma_{i}, \delta_{i}\right)+\left.\partial_{l} u_{h}\right|_{K_{i+1}}\left(\gamma_{i}, \delta_{i}\right)\right]\right| \\
\leq C h^{2}|\ln h|\|u\|_{3, \infty, \Omega}, \quad i=1, \ldots, 6
\end{gathered}
$$

Since $u_{h}$ is piecewise linear on $\omega_{0}=\cup_{i=1}^{6} K_{i}$, we have

$$
\sum_{i=1}^{6} \frac{1}{2}\left[\left.\partial_{l} u_{h}\right|_{K_{i}}\left(\gamma_{i}, \delta_{i}\right)+\left.\partial_{l} u_{h}\right|_{K_{i+1}}\left(\gamma_{i}, \delta_{i}\right)\right]=\sum_{i=1}^{6} \partial_{l} u_{h}\left(\xi_{i}, \eta_{i}\right)
$$


which together with (17) leads to

$$
\left|\sum_{i=1}^{6}\left[\partial_{l} u\left(\gamma_{i}, \delta_{i}\right)-\partial_{l} u_{h}\left(\xi_{i}, \eta_{i}\right)\right]\right| \leq C h^{2}|\ln h|\|u\|_{3, \infty, \Omega} .
$$

By the Taylor expansion we have

$$
\begin{gathered}
\partial_{l} u\left(\gamma_{i}, \delta_{i}\right)=\partial_{l} u\left(\alpha_{0}, \beta_{0}\right)+\nabla \partial_{l} u\left(\alpha_{0}, \beta_{0}\right) \cdot\left[\left(\gamma_{i}, \delta_{i}\right)-\left(\alpha_{0}, \beta_{0}\right)\right]+R_{i}, \\
\left|R_{i}\right| \leq C h^{2}\|u\|_{3, \infty, \omega_{0}}, \quad i=1, \ldots, 6 .
\end{gathered}
$$

Since $\tau_{h}$ is strongly regular, we also have

$$
\left|\sum_{i=1}^{6}\left[\left(\gamma_{i}, \delta_{i}\right)-\left(\alpha_{0}, \beta_{0}\right)\right]\right| \leq C h^{2} .
$$

Consequently, we have by (18) that

$$
\begin{aligned}
& \left|\partial_{l} u\left(\alpha_{0}, \beta_{0}\right)-\frac{1}{6} \sum_{i=1}^{6} \partial_{l} u_{h}\left(\xi_{i}, \eta_{i}\right)\right| \\
& \leq \frac{1}{6}\left|\sum_{i=1}^{6}\left[\partial_{l} u\left(\gamma_{i}, \delta_{i}\right)-\partial_{l} u_{h}\left(\xi_{i}, \eta_{i}\right)\right]\right| \\
& \quad+\frac{1}{6}\left|\nabla \partial_{l} u\left(\alpha_{0}, \beta_{0}\right)\right|\left|\sum_{i=1}^{6}\left[\left(\gamma_{i}, \delta_{i}\right)-\left(\alpha_{0}, \beta_{0}\right)\right]\right|+\frac{1}{6} \sum_{i=1}^{6}\left|R_{i}\right| \\
& \leq C h^{2}|\ln h|\|u\|_{3, \infty, \Omega} .
\end{aligned}
$$

Let $F_{l}: P\left(\omega_{0}\right) \rightarrow \mathbf{R}$ be the functional as defined by (10) for the locally discrete least-squares recovery and as defined by (11) for the local $L^{2}$ projection recovery. Since $p_{l} \in P_{1}\left(\omega_{0}\right)$ is the unique minimizer of $F_{l}$ by the assumption of the theorem, it satisfies the corresponding equation (5) or (6).

We now consider two cases.

Case 1. The locally discrete least-squares recovery. Taking $q=1$ identically on $\omega_{0}$ in (5) with $m=6$ and $P=P_{1}$, we obtain in this case that

$$
\sum_{i=1}^{6} p_{l}\left(\xi_{i}, \eta_{i}\right)=\sum_{i=1}^{6} \partial_{l} u_{h}\left(\xi_{i}, \eta_{i}\right)
$$

By the strongly regular property of the triangular mesh $\tau_{h}$, we have

$$
\left|\sum_{i=1}^{6}\left[\left(\xi_{i}, \eta_{i}\right)-\left(\alpha_{0}, \beta_{0}\right)\right]\right| \leq C h^{2} .
$$


Since $p_{l}$ is linear on $\omega_{0}$ in this case, we thus conclude that

$$
\begin{aligned}
& \left|p_{l}\left(\alpha_{0}, \beta_{0}\right)-\frac{1}{6} \sum_{i=6}^{6} p_{l}\left(\xi_{i}, \eta_{i}\right)\right| \\
& \quad=\frac{1}{6}\left|\sum_{i=1}^{6}\left[p_{l}\left(\alpha_{0}, \beta_{0}\right)-p_{l}\left(\xi, \eta_{i}\right)\right]\right| \\
& \quad=\frac{1}{6}\left|\nabla p_{l}\left(\alpha_{0}, \beta_{0}\right) \cdot \sum_{i=1}^{6}\left[\left(\xi_{i}, \eta_{i}\right)-\left(\alpha_{0}, \beta_{0}\right)\right]\right| \\
& \quad \leq C h^{2}\left|\nabla p_{l}\left(\alpha_{0}, \beta_{0}\right)\right|
\end{aligned}
$$

which together with (12), (20) and (19) implies (7) in this case.

Case 2. The local $L^{2}$ projection recovery. Since $p_{l}$ is linear on $\omega_{0}$, we have by taking $q=1$ identically on $\omega_{0}$ in (6) with $P=P_{1}$ that

$$
\sum_{i=1}^{6}\left|K_{i}\right| p_{l}\left(\xi_{i}, \eta_{i}\right)=\sum_{i=1}^{6}\left|K_{i}\right| \partial_{l} u_{h}\left(\xi_{i}, \eta_{i}\right),
$$

where we denote by $|\omega|$ the measure of a measurable set $\omega \subset \bar{\Omega}$.

By the strongly regular property of the mesh $\tau_{h}$, we deduce easily that

$$
|| K_{i}\left|-\frac{1}{6}\right| \omega_{0}|| \leq C h^{3}, \quad i=1, \ldots, 6 .
$$

By the Taylor expansion we have

$$
\begin{aligned}
\partial_{l} u\left(\xi_{i}, \eta_{i}\right)= & \partial_{l} u\left(\alpha_{0}, \beta_{0}\right)+\nabla \partial_{l} u\left(\alpha_{0}, \beta_{0}\right) \cdot\left(\xi_{i}-\alpha_{0}, \eta_{i}-\beta_{0}\right)+S_{i}, \\
& \left|S_{i}\right| \leq C h^{2}\|u\|_{3, \infty, \omega_{0}}, \quad i=1, \ldots, 6 .
\end{aligned}
$$

Consequently, by the fact that $\sum_{i=1}^{6}\left|K_{i}\right|=\left|\omega_{0}\right|$, we get

$$
\begin{aligned}
& \left|\sum_{i=1}^{6}\left(\left|K_{i}\right|-\frac{1}{6}\left|\omega_{0}\right|\right) \partial_{l} u\left(\xi_{i}, \eta_{i}\right)\right| \\
& \quad=\left|\sum_{i=1}^{6}\left(\left|K_{i}\right|-\frac{1}{6}\left|\omega_{0}\right|\right)\left[\partial_{l} u\left(\alpha_{0}, \beta_{0}\right)+\nabla \partial_{l} u\left(\alpha_{0}, \beta_{0}\right) \cdot\left(\xi_{i}-\alpha_{0}, \eta_{i}-\beta_{0}\right)+S_{i}\right]\right| \\
& \quad=\left|\sum_{i=1}^{6}\left(\left|K_{i}\right|-\frac{1}{6}\left|\omega_{0}\right|\right)\left[\nabla \partial_{l} u\left(\alpha_{0}, \beta_{0}\right) \cdot\left(\xi_{i}-\alpha_{0}, \eta_{i}-\beta_{0}\right)+S_{i}\right]\right| \\
& \quad \leq C h^{4}\|u\|_{3, \infty, \omega_{0}} .
\end{aligned}
$$


We thus have by (23) and the $W^{1, \infty}$ estimate for the linear finite element approximation $[6,9,20,21,22,32]$ that

$$
\begin{aligned}
& \left|\sum_{i=1}^{6}\left(\left|K_{i}\right|-\frac{1}{6}\left|\omega_{0}\right|\right) \partial_{l} u_{h}\left(\xi_{i}, \eta_{i}\right)\right| \\
& \leq\left|\sum_{i=1}^{6}\left(\left|K_{i}\right|-\frac{1}{6}\left|\omega_{0}\right|\right)\left[\partial_{l} u_{h}\left(\xi_{i}, \eta_{i}\right)-\partial_{l} u\left(\xi_{i}, \eta_{i}\right)\right]\right| \\
& \quad+\left|\sum_{i=1}^{6}\left(\left|K_{i}\right|-\frac{1}{6}\left|\omega_{0}\right|\right) \partial_{l} u\left(\xi_{i}, \eta_{i}\right)\right| \\
& \leq C h^{4}\|u\|_{3, \infty, \Omega} .
\end{aligned}
$$

This together with (19) leads to

$$
\begin{aligned}
|| \omega_{0}\left|\partial_{l} u\left(\alpha_{0}, \beta_{0}\right)-\sum_{i=1}^{6}\right| K_{i}\left|\partial_{l} u_{h}\left(\xi_{i}, \eta_{i}\right)\right| \\
\leq|| \omega_{0}\left|\partial_{l} u\left(\alpha_{0}, \beta_{0}\right)-\frac{1}{6}\right| \omega_{0}\left|\sum_{i=1}^{6} \partial_{l} u_{h}\left(\xi_{i}, \eta_{i}\right)\right| \\
\quad+\left|\sum_{i=1}^{6}\left(\left|K_{i}\right|-\frac{1}{6}\left|\omega_{0}\right|\right) \partial_{l} u_{h}\left(\xi_{i}, \eta_{i}\right)\right| \\
\leq C h^{4}|\ln h|\|u\|_{3, \infty, \Omega} .
\end{aligned}
$$

Finally, we have by (23), (21) and the stability estimate (12) that

$$
\begin{aligned}
& || \omega_{0}\left|p_{l}\left(\alpha_{0}, \beta_{0}\right)-\sum_{i=1}^{6}\right| K_{i}\left|p_{l}\left(\xi_{i}, \eta_{i}\right)\right| \\
& =|| \omega_{0}\left|p_{l}\left(\alpha_{0}, \beta_{0}\right)-\sum_{i=1}^{6}\right| K_{i}\left|\left[p_{l}\left(\alpha_{0}, \beta_{0}\right)+\nabla p_{l}\left(\alpha_{0}, \beta_{0}\right) \cdot\left(\xi_{i}-\alpha_{0}, \eta_{i}-\beta_{0}\right)\right]\right| \\
& =\left|\nabla p_{l}\left(\alpha_{0}, \beta_{0}\right) \cdot \sum_{i=1}^{6}\right| K_{i}\left|\left(\xi_{i}-\alpha_{0}, \eta_{i}-\beta_{0}\right)\right| \\
& \leq\left|\nabla p_{l}\left(\alpha_{0}, \beta_{0}\right)\right|\left|\sum_{i=1}^{6}\left(\left|K_{i}\right|-\frac{1}{6}\left|\omega_{0}\right|\right)\left(\xi_{i}-\alpha_{0}, \eta_{i}-\beta_{0}\right)\right| \\
& \quad+\frac{1}{6}\left|\nabla p_{l}\left(\alpha_{0}, \beta_{0}\right)\right|\left|\omega_{0}\right|\left|\sum_{i=1}^{6}\left(\xi_{i}-\alpha_{0}, \eta_{i}-\beta_{0}\right)\right|
\end{aligned}
$$




$$
\begin{aligned}
& \leq C h^{4}\left|\nabla p_{l}\left(\alpha_{0}, \beta_{0}\right)\right| \\
& \leq C h^{4}\|u\|_{3, \infty, \Omega},
\end{aligned}
$$

which together with (22), (24), and the quasi-uniformity of the mesh implies (7) in this case. The proof is complete.

Proof of Theorem 2 Let $u,\left(\alpha_{0}, \beta_{0}\right), K_{i}$, and $\omega_{0}=\cup_{i=1}^{m} K_{i}$ be all the same as in the theorem. Since the mesh $\tau_{h}$ is a rectangular mesh, we have $m=4$. Denoting again by $\left(\xi_{i}, \eta_{i}\right)$ the center of the element $K_{i}, i=1, \ldots, 4$, we recall the superconvergence estimate in the present setting from the previous work $[6,10,11,13,23,32,37]$

$$
\left|\partial_{l} u\left(\xi_{i}, \eta_{i}\right)-\partial_{l} u_{h}\left(\xi_{i}, \eta_{i}\right)\right| \leq C h^{2}|\ln h|\|u\|_{3, \infty, \Omega}, \quad i=1, \ldots, 4 .
$$

Fix $l=1$ or 2 . Let $F_{l}: P\left(\omega_{0}\right) \rightarrow \mathbf{R}$ be the functional as defined by (10) for the locally discrete least-squares recovery and as defined by (11) for the local $L^{2}$ projection recovery. Since $p_{l} \in P_{1}\left(\omega_{0}\right)$ is the unique minimizer of $F_{l}$ by the assumption of the theorem, it satisfies the corresponding equation (5) or $(6)$.

We now consider two cases.

Case 1. The locally discrete least-squares recovery on a quasi-uniform rectangular mesh. For convenience, we number all the four elements in the element patch $\omega_{0}$ counterclockwise starting from the lower left one. Define $q_{i} \in Q_{1}\left(\omega_{0}\right)$ for $i=1, \ldots, 4$ by

$$
q_{i}(x, y)=(-1)^{i+1} \frac{4}{\left|\omega_{0}\right|}\left(x-\xi_{i+2}\right)\left(y-\eta_{i+2}\right), \quad(x, y) \in \omega_{0},
$$

where we adopt the convention that two indices $i_{1}$ and $i_{2}$ are the same if $i_{1} \equiv i_{2}(\bmod 4)$. Since the four centers $\left(\xi_{i}, \eta_{i}\right) \in K_{i}, i=1, \ldots, 4$, are vertices of a rectangle with its sides parallel to the coordinate axes, respectively, it is easy to verify that $q_{i}\left(\xi_{j}, \eta_{j}\right)=\delta_{i j}, i, j=1, \ldots, 4$. So, taking $q=q_{j}$ in (5) with $m=4$ and $P=Q_{1}$, we get

$$
p_{l}\left(\xi_{j}, \eta_{j}\right)=\partial_{l} u_{h}\left(\xi_{j}, \eta_{j}\right), \quad j=1, \ldots, 4 .
$$

Let $h_{x i}$ and $h_{y i}$ be the length of sides of $K_{i}$ along $O x$ and $O y$ coordinate directions, respectively, i.e.,

$$
h_{x i}=2\left|\alpha_{0}-\xi_{i}\right|, \quad h_{y i}=2\left|\beta_{0}-\eta_{i}\right|, \quad i=1, \ldots, 4 .
$$


We have by an easy calculation that

$$
q_{i}\left(\alpha_{0}, \beta_{0}\right)=\frac{h_{x i+2} h_{y i+2}}{\left|\omega_{0}\right|}, \quad i=1, \ldots, 4 .
$$

Therefore,

$$
\begin{aligned}
q\left(\alpha_{0}, \beta_{0}\right) & =\sum_{i=1}^{4} q\left(\xi_{i}, \eta_{i}\right) q_{i}\left(\alpha_{0}, \beta_{0}\right) \\
& =\frac{1}{\left|\omega_{0}\right|} \sum_{i=1}^{4} h_{x i+2} h_{y i+2} q\left(\xi_{i}, \eta_{i}\right), \quad \forall q \in Q_{1}\left(\omega_{0}\right) .
\end{aligned}
$$

Now let $B_{l} \in Q_{1}\left(\omega_{0}\right)$ be the bilinear part of $\partial_{l} u$ on $\omega_{0}$ around the assembly point $\left(\alpha_{0}, \beta_{0}\right)$ :

$$
\begin{aligned}
B_{l}(x, y)=\partial_{l} u & \left(\alpha_{0}, \beta_{0}\right)+\nabla \partial_{l} u\left(\alpha_{0}, \beta_{0}\right) \cdot\left(x-\alpha_{0}, y-\beta_{0}\right) \\
& +\partial_{1} \partial_{2} \partial_{l} u\left(\alpha_{0}, \beta_{0}\right)\left(x-\alpha_{0}\right)\left(y-\beta_{0}\right), \quad(x, y) \in \omega_{0} .
\end{aligned}
$$

Obviously, $B_{l}\left(\alpha_{0}, \beta_{0}\right)=\partial_{l} u\left(\alpha_{0}, \beta_{0}\right)$, and

$$
\left|B_{l}(x, y)-\partial_{l} u(x, y)\right| \leq C h^{2}\|u\|_{3, \infty, \omega_{0}}, \quad \forall(x, y) \in \omega_{0} .
$$

Therefore, using the expression (27) for $p_{l}$ and $B_{l}$, respectively, we have by (26), (25), and (28) that

$$
\begin{aligned}
& \left|p_{l}\left(\alpha_{0}, \beta_{0}\right)-\partial_{l} u\left(\alpha_{0}, \beta_{0}\right)\right| \\
& \quad=\left|p_{l}\left(\alpha_{0}, \beta_{0}\right)-B_{l} u\left(\alpha_{0}, \beta_{0}\right)\right| \\
& \quad=\frac{1}{\left|\omega_{0}\right|}\left|\sum_{i=1}^{4} h_{x i+2} h_{y i+2}\left[p_{l}\left(\xi_{i}, \eta_{i}\right)-B_{l}\left(\xi_{i}, \eta_{i}\right)\right]\right| \\
& \quad \leq \sum_{i=1}^{4}\left|\partial_{l} u_{h}\left(\xi_{i}, \eta_{i}\right)-\partial_{l} u\left(\xi_{i}, \eta_{i}\right)\right|+\sum_{i=1}^{4}\left|\partial_{l} u\left(\xi_{i}, \eta_{i}\right)-B_{l}\left(\xi_{i}, \eta_{i}\right)\right| \\
& \quad \leq C h^{2}|\ln h|\|u\|_{3, \infty, \Omega},
\end{aligned}
$$

proving (8) in this case.

Case 2. The local $L^{2}$ projection recovery on a unidirectionally uniform rectangular mesh. We have in this special case that $\left|K_{i}\right|=\frac{1}{4}\left|\omega_{0}\right|$ for $i=$ 
$1, \ldots, 4$. Setting $q=1$ identically on $\omega_{0}$ in (6) with $P=Q_{1}$, by the fact that $p_{l}$ is bilinear and $u_{h}$ piecewise bilinear, we obtain that

$$
\sum_{i=1}^{4} p_{l}\left(\xi_{i}, \eta_{i}\right)=\sum_{i=1}^{4} \partial_{l} u_{h}\left(\xi_{i}, \eta_{i}\right)
$$

By a simple calculation we also have that

$$
q\left(\alpha_{0}, \beta_{0}\right)=\frac{1}{4} \sum_{i=1}^{4} q\left(\xi_{i}, \eta_{i}\right), \quad \forall q \in Q_{1}\left(\omega_{0}\right) .
$$

It therefore follows from (29), (28) and (25) that

$$
\begin{aligned}
& \left|p_{l}\left(\alpha_{0}, \beta_{0}\right)-\partial_{l} u\left(\alpha_{0}, \beta_{0}\right)\right| \\
& \quad=\left|p_{l}\left(\alpha_{0}, \beta_{0}\right)-B_{l}\left(\alpha_{0}, \beta_{0}\right)\right| \\
& \quad=\frac{1}{4}\left|\sum_{i=1}^{4} p_{l}\left(\xi_{i}, \eta_{i}\right)-\sum_{i=1}^{4} B_{l}\left(\xi_{i}, \eta_{i}\right)\right| \\
& \quad \leq \frac{1}{4}\left|\sum_{i=1}^{4} \partial_{l} u_{h}\left(\xi_{i}, \eta_{i}\right)-\sum_{i=1}^{4} B_{l}\left(\xi_{i}, \eta_{i}\right)\right| \\
& \quad \leq \frac{1}{4} \sum_{i=1}^{4}\left|\partial_{l} u_{h}\left(\xi_{i}, \eta_{i}\right)-\partial_{l} u\left(\xi_{i}, \eta_{i}\right)\right|+\frac{1}{4} \sum_{i=1}^{4}\left|\partial_{l} u\left(\xi_{i}, \eta_{i}\right)-B_{l}\left(\xi_{i}, \eta_{i}\right)\right| \\
& \quad \leq C h^{2}|\ln h|\|u\|_{3, \infty, \Omega},
\end{aligned}
$$

which implies (8) in this case. The proof is finished.

Proof of Theorem 3 and Theorem 4 It follows from the definition of $W^{-1,2}(\Omega)$-norm and the standard $L^{2}$ error estimate that

$$
\left\|u-u_{h}\right\|_{-1,2, \Omega} \leq\left\|u-u_{h}\right\|_{0,2, \Omega} \leq C h^{2}\|u\|_{2,2, \Omega} .
$$

Therefore, under the assumption of the theorems, we have the local $W^{1, \infty}$ estimate $[6,22,23,32]$

$$
\left\|u-u_{h}\right\|_{1, \infty, \Omega_{0}} \leq C h\left(\|u\|_{2, \infty, \Omega_{1}}+\|u\|_{2,2, \Omega}\right) .
$$

Since the element patch $\omega_{0}=\cup_{i=1}^{m} K_{i} \subset \bar{\Omega}_{0}$, the stability estimate (12) becomes now (cf. (14) and (16))

$$
\left|\nabla p_{l}\left(\alpha_{0}, \beta_{0}\right)\right| \leq C\left(\|u\|_{3, \infty, \Omega_{1}}+\|u\|_{2,2, \Omega}\right), \quad l=1,2 .
$$


Further, the superconvergence estimates (17) and (25) become respectively $[6,22,23,24,31,32]$

$$
\begin{aligned}
& \left|\partial_{l} u\left(\gamma_{i}, \delta_{i}\right)-\frac{1}{2}\left[\left.\partial_{l} u_{h}\right|_{K_{i}}\left(\gamma_{i}, \delta_{i}\right)+\left.\partial_{l} u_{h}\right|_{K_{i+1}}\left(\gamma_{i}, \delta_{i}\right)\right]\right| \\
& \quad \leq C h^{2}|\ln h|\left(\|u\|_{3, \infty, \Omega_{1}}+\|u\|_{2,2, \Omega}\right), \quad i=1, \ldots, 6,
\end{aligned}
$$

and

$$
\begin{aligned}
& \left|\partial_{l} u\left(\xi_{i}, \eta_{i}\right)-\partial_{l} u_{h}\left(\xi_{i}, \eta_{i}\right)\right| \\
& \quad \leq C h^{2}|\ln h|\left(\|u\|_{3, \infty, \Omega_{1}}+\|u\|_{2,2, \Omega}\right), \quad i=1, \ldots, 4
\end{aligned}
$$

Using (30) - (33), we can obtain the desired local superconvergence estimates by repeating the corresponding proofs of Theorem 1 and Theorem 2 .

\section{An Example}

In this section, we give a simple example to show that in the one-dimensional case the quasi-uniformity of a mesh is in general not sufficient to result in the derivative superconvergence by the local $L^{2}$ projection recovery. It also strongly suggests that in the two-dimensional case the derivative superconvergence will not be recovered in general by the rectangular bilinear element using the local $L^{2}$ projection if the underlying rectangular mesh is only quasiuniform, cf., the numerical experiments reported in [34].

We consider the two-point boundary value problem

$$
\left\{\begin{array}{l}
-u^{\prime \prime}=f, \quad \text { in }(0,1), \\
u(0)=u(1)=0,
\end{array}\right.
$$

where $f \in L^{2}(0,1)$. Let $u$ be the exact solution of (34) and assume that $u$ is smooth enough on $[0,1]$.

Let $\tau_{h}: 0=x_{0}<x_{1}<\cdots<x_{N}=1$ be an arbitrary mesh of the interval $[0,1]$, where $h=\max \left\{h_{i}: 1 \leq i \leq N\right\}$ and $h_{i}=x_{i}-x_{i-1}, i=1, \ldots, N$.

Denote by $V_{h}$ the corresponding linear finite element space:

$$
V_{h}=\left\{v_{h} \in H_{0}^{1}(0,1):\left.v_{h}\right|_{\left(x_{i-1}, x_{i}\right)} \in P_{1}\left(x_{i-1}, x_{i}\right), i=1, \ldots, N\right\},
$$

where $P_{1}$ denotes the space of all one-variable, linear polynomials. Let $u_{h} \in$ $V_{h}$ be the finite element approximation of the exact solution $u$ defined by

$$
\int_{0}^{1}\left(u^{\prime}-u_{h}^{\prime}\right) v_{h}^{\prime} d x=0, \quad \forall v_{h} \in V_{h} .
$$


Let $I_{h} u \in V_{h}$ be the Lagrange interpolant of $u$ defined by

$$
I_{h} u\left(x_{i}\right)=u\left(x_{i}\right), \quad i=0,1, \ldots, N .
$$

For any $v_{h} \in V_{h}$, we have by (35) that

$$
\begin{aligned}
\int_{0}^{1} & \left(u_{h}-I_{h} u\right)^{\prime} v_{h}^{\prime} d x=\int_{0}^{1}\left(u-I_{h} u\right)^{\prime} v_{h}^{\prime} d x \\
& =\sum_{i=1}^{N} \int_{x_{i-1}}^{x_{i}}\left(u-I_{h} u\right)^{\prime} v_{h}^{\prime} d x=\left.\sum_{i=1}^{N}\left(u-I_{h} u\right) v_{h}^{\prime}\right|_{x_{i-1}} ^{x_{i}}=0 .
\end{aligned}
$$

Setting $v_{h}=u_{h}-I_{h} u$, we obtain by the constraint of zero boundary value that in this case $u_{h}=I_{h} u$ identically on [0,1], a well-known result.

Now we consider an element patch $\omega_{i}=\left(x_{i-1}, x_{i+1}\right)$ surrounding the assembly point $x_{i}(1 \leq i \leq N-1)$. Let $p \in P_{1}\left(x_{i-1}, x_{i+1}\right)$ be the recovered derivative by the local $L^{2}$ projection which is determined by (cf. Lemma 1)

$$
\int_{x_{i-1}}^{x_{i+1}}\left(p-u_{h}^{\prime}\right) q d x=0, \quad \forall q \in P_{1}\left(x_{i-1}, x_{i+1}\right) .
$$

Assuming that $p(x)=a_{0}+a_{1}\left(x-x_{i}\right)$ for $x \in\left(x_{i-1}, x_{i+1}\right)$, where $a_{0}$ and $a_{1}$ are constants, we obtain since $u_{h}=I_{h} u$ that

$$
\int_{x_{i-1}}^{x_{i+1}}\left[a_{0}+a_{1}\left(x-x_{i}\right)-\left(I_{h} u\right)^{\prime}\right] q d x=0, \quad \forall q \in P_{1}\left(x_{i-1}, x_{i+1}\right) .
$$

Setting $q(x)=1$ and $q(x)=x-x_{i}$, respectively, we have by a series of calculations that

$$
\begin{aligned}
\left(h_{i}+h_{i+1}\right) a_{0}+\frac{1}{2}\left(h_{i+1}^{2}-h_{i}^{2}\right) a_{1} & =u_{i+1}-u_{i-1}, \\
\frac{1}{2}\left(h_{i+1}^{2}-h_{i}^{2}\right) a_{0}+\frac{1}{3}\left(h_{i}^{3}+h_{i+1}^{3}\right) a_{1} & =\frac{1}{2} h_{i+1}\left(u_{i+1}-u_{i}\right)-\frac{1}{2} h_{i}\left(u_{i}-u_{i-1}\right),
\end{aligned}
$$

where we recall $h_{j}=x_{j}-x_{j-1}, j=1, \ldots, N$, and we denote $u_{j}=u\left(x_{j}\right)$ for $j=0,1, \ldots, N$.

Notice that $p\left(x_{i}\right)=a_{0}$. So, we need only to find $a_{0}$. Solving the above two equations, we obtain that

$$
\begin{gathered}
a_{0}=\frac{1}{\left(h_{i}+h_{i+1}\right)^{3}}\left[\left(u_{i+1}-u_{i}\right)\left(4 h_{i}^{2}+h_{i+1}^{2}-h_{i} h_{i+1}\right)\right. \\
\left.\quad+\left(u_{i}-u_{i-1}\right)\left(4 h_{i+1}^{2}+h_{i}^{2}-h_{i} h_{i+1}\right)\right] .
\end{gathered}
$$


By the Taylor expansion we have

$$
u_{i+1}=u_{i}+h_{i+1} u^{\prime}\left(x_{i}\right)+\frac{1}{2} h_{i+1}^{2} u^{\prime \prime}\left(x_{i}\right)+\frac{1}{6} h_{i+1}^{3} u^{\prime \prime \prime}\left(\bar{x}_{i+1}\right)
$$

for some $\bar{x}_{i+1} \in\left[x_{i}, x_{i+1}\right]$, and

$$
u_{i-1}=u_{i}-h_{i} u^{\prime}\left(x_{i}\right)+\frac{1}{2} h_{i}^{2} u^{\prime \prime}\left(x_{i}\right)-\frac{1}{6} h_{i}^{3} u^{\prime \prime \prime}\left(\bar{x}_{i}\right)
$$

for some $\bar{x}_{i} \in\left[x_{i-1}, x_{i}\right]$. Consequently, we have by a series of calculations that

$$
\begin{aligned}
p\left(x_{i}\right)-u^{\prime}\left(x_{i}\right) & =a_{0}-u^{\prime}\left(x_{i}\right) \\
& =\frac{h_{i}^{3}+h_{i+1}^{3}}{2\left(h_{i}+h_{i+1}\right)^{3}}\left(h_{i+1}-h_{i}\right) u^{\prime \prime}\left(x_{i}\right)+r_{i}
\end{aligned}
$$

with

$$
\left|r_{i}\right| \leq \frac{1}{6}\left(h_{i}+h_{i+1}\right)^{2} \max _{x_{i-1} \leq x \leq x_{i+1}}\left|u^{\prime \prime \prime}(x)\right| .
$$

It is easy to verify that

$$
\frac{1}{8} \leq\left|\frac{h_{i}^{3}+h_{i+1}^{3}}{2\left(h_{i}+h_{i+1}\right)^{3}}\right| \leq \frac{1}{2} .
$$

Therefore, to recover the derivative superconvergence at all assembly points $x_{i}$ by the local $L^{2}$ projection, we need to have the condition that $\left|h_{i+1}-h_{i}\right|=$ $O\left(h^{2}\right)$ for all $i=1, \ldots, N-1$. This is, however, certainly much stronger than the quasi-uniformity condition which only guarantees in general that $\left|h_{i+1}-h_{i}\right|=O(h)$ for all $i=1, \ldots, N-1$.

Acknowledgment. The authors thank Dr. J. Z. Zhu for drawing their attention to the subject of mathematical analysis on the Zienkiewicz-Zhu discrete least-squares based superconvergence patch recovery technique.

\section{References}

[1] I. Babuška, T. Strouboulis, S. K. Gangaraj, and C. S. Upadhyay. $\eta \%$ superconvergence in the interior of locally refined meshes of quadrilaterals: Superconvergence of the gradient in finite element solutions of Laplace's and Poisson's equations. Appl. Numer. Math., 16:3-49, 1994. 
[2] I. Babuška, T. Strouboulis, S. K. Gangaraj, and C. S. Upadhyay. Validation of recipes for the recovery of stresses and derivatives by a computerbased approach. Math. Comput. Modelling, 20:45-89, 1994.

[3] I. Babuška, T. Strouboulis, and C. S. Upadhyay. $\eta \%$-superconvergence of finite element approximations in the interior of general meshes of triangles. Comput. Methods Appl. Mech. Engrg., 122:273-305, 1995.

[4] I. Babuška, T. Strouboulis, C. S. Upadhyay, and S. K. Gangaraj. Computer-based proof of the existence of superconvergence points in the finite element method: superconvergence of derivatives in finite element solutions of Laplace's, Poisson's, and the elasticity equations. Numer. Methods for PDEs, 12:347-392, 1996.

[5] C. M. Chen. Optimal points of the stresses for triangular linear elements. Numer. Math. J. Chinese Univ., 2:12-20, 1980 (in Chinese).

[6] C. M. Chen and Y. Q. Huang. High Accuracy Theory of Finite Element Methods. Hunan Science Press, Hunan, China, 1995 (in Chinese).

[7] H. S. Chen and B. Li. Superconvergence analysis and error expansion for the Wilson nonconforming finite element. Numer. Math., 69:125-140, 1994.

[8] H. S. Chen and Q. Lin. Extrapolation for isoparametric bilinear finite element approximation on smooth bounded domains. J. Syst. Sci. Math., 2:127-140, 1992 .

[9] P. G. Ciarlet. Basic error estimates for elliptic problems. In P. G. Ciarlet and J. L. Lions, editors, Handbook of Numerical Analysis, Vol. II, Finite element methods (Part 1), pages 17-351. Elsevier Science Publishers, 1991.

[10] M. Křrižek and P. Neittaanmäki. On superconvergence techniques. Acta Appl. Math., 9:175-198, 1987.

[11] P. Lesaint and M. Zlámal. Superconvergence of the gradient of finite element solutions. RAIRO Modél. Math. Anal. Numér., 13:139-166, 1979 . 
[12] N. D. Levine. Superconvergent recovery of the gradient from piecewise linear finite element approximations. IMA J. Numer. Anal., 5:407-427, 1985 .

[13] Q. Lin and J. R. Whiteman. Superconvergence of recovered gradients of finite element approximations on nonuniform rectangular and quadrilateral meshes. In J. R. Whiteman, editor, The Mathematics of Finite Elements and Applications VII, pages 563-571. Academic Press, 1991.

[14] Q. Lin and J. Xu. Linear finite elements with high accuracy. J. Comp. Math., 3:115-133, 1985.

[15] Q. Lin, N. Yan, and A. Zhou. A rectangle test for interpolated finite elements. In Proc. of Sys. Sci. \& Sys. Eng., pages 217-229, Hong Kong, 1991. Great Wall Culture Publish. Co.

[16] Q. Lin and Y. D. Yang. Interpolation and correction of finite elements. Math. Practice \& Theory, 3:17-28, 1991 (in Chinese).

[17] Q. Lin and Y. D. Yang. The finite element interpolated correction method for elliptic eigenvalue problems. Math. Numer. Sinica, 3:334338, 1992 (in Chinese).

[18] Q. Lin and A. Zhou. Notes on superconvergence and its related topics. J. Comp. Math., 11(3):211-214, 1993.

[19] Q. Lin and Q. D. Zhu. The Preprocessing and Postprocessing for the Finite Element method. Shanghai Scientific \& Technical Publishers, Shanghai, China, 1994 (in Chinese).

[20] J. A. Nitsche. $L_{\infty}$-convergence of finite element approximations. In I. Galligani and E. Magenes, editors, Mathematical Aspects of Finite Element Methods, volume 606 of Lecture Notes in Mathematics, pages 261-274, Springer, Berlin, 1977.

[21] L. R. Scott. Optimal $l^{\infty}$ estimates for the finite element method on irregular meshes. Math. Comp., 30:681-697, 1976.

[22] L. B. Wahlbin. Local behavior in finite element methods. In P. G. Ciarlet and J. L. Lions, editors, Handbook of Numerical Analysis, Vol. II, Finite 
element methods (Part 1), pages 353-522. Elsevier Science Publishers, 1991.

[23] L. B. Wahlbin. Superconvergence in Galerkin Finite Element Methods, volume 1605 of Lecture Notes in Mathematics. Springer, Berlin, 1995.

[24] M. F. Wheeler and J. R. Whiteman. Superconvergent recovery of gradients on subdomains from piecewise linear finite element approximations. Numer. Methods for PDEs, 3:65-82, 1987.

[25] Z. Zhang. Ultraconvergence of the patch recovery technique. Math. Comp., 65:1431-1437, 1996.

[26] Z. Zhang and H. D. Victory Jr. Mathematical analysis of ZienkiewiczZhu's derivative patch recovery technique for quadrilateral finite elements. Numer. Methods for PDEs., 12:507-524, 1996.

[27] Z. Zhang and J. Z. Zhu. Analysis of the superconvergent patch recovery technique and a posteriori error estimator in the finite element method (I). Comput. Methods Appl. Mech. Engrg., 123:173-187, 1995.

[28] Z. Zhang and J. Z. Zhu. Superconvergence of the derivative patch recovery technique and a posteriori error estimation. In I. Babuška, J. E. Flaherty, J. E. Hopcroft, W. D. Henshaw, J. E. Oliger, and T. Tezduyar, editors, Modeling, mesh generation, and adaptive numerical methods for partial differential equations, pages 431-450, Minneapolis, 1995. Springer-Verlag, New York.

[29] A. Zhou. Global superconvergence approximations of the mixed finite element method for the stokes problem and the linear elasticity equation. Math. Modeling and Numer. Anal., 30(4):401-411, 1996.

[30] A. Zhou and J. Li. The full approximation accuracy for the stream function-vorticity-pressure method. Numer. Math., 68:427-435, 1994.

[31] Q. D. Zhu. Natural inner superconvergence for the finite element method. In K. Feng and J. L. Lions, editors, Proceedings of ChinaFrance Symposium on Finite Element Methods, pages 935-960, Beijing, 1983. Gordon and Breach, New York. 
[32] Q. D. Zhu and Q. Lin. Superconvergence Theory of the Finite Element Method. Hunan Science Press, Hunan, China, 1989 (in Chinese).

[33] O. C. Zienkiewicz and J. Z. Zhu. A simple error estimator and the adaptive procedure for practical engineering analysis. Int. J. Numer. Methods Eng., 24:337-357, 1987.

[34] O. C. Zienkiewicz and J. Z. Zhu. The superconvergence patch recovery and a posteriori error estimates. Part 1: The recovery technique. Int. J. Numer. Methods Engrg., 33:1331-1364, 1992.

[35] O. C. Zienkiewicz and J. Z. Zhu. The superconvergence patch recovery and a posteriori error estimates. Part 2: Error estimates and adaptivity. Int. J. Numer. Methods Engrg., 33:1365-1382, 1992.

[36] O. C. Zienkiewicz and J. Z. Zhu. The superconvergence patch recovery (SPR) and adaptive finite element refinement. Comput. Methods Appl. Mech. Engrg., 101:207-224, 1992.

[37] M. Zlámal. Superconvergence and reduced integration in the finite element method. Math. Comp., 32:663-685, 1978. 